\title{
A helical hydrogen-MARFE-like phenomenon in the divertor of the Wendelstein 7-AS stellarator
}

\author{
U. Wenzel, R. König, T. Sunn Pedersen and the W7-AS \\ team \\ Max Planck Institute for Plasma Physics, \\ Wendelsteinstr. 1, 17491 Greifswald, Germany
}

\begin{abstract}
In the island divertor of the W7-AS stellarator a high-density zone (HDZ) near the divertor plates was discovered some years ago [1] with electron densities up to $7 * 10^{20} \mathrm{~m}^{-3}$. We shed further light on this phenomenon by determining the poloidal and radial location of this zone and discussing potential implications of these findings. The high-density zone is in the vicinity of, but clearly separated from the nearest X-point line. The carbon emission is clearly spatially separated, residing near or at the x-point lines. The HDZ shows many similarities with the hydrogen or wall MARFE in Textor-94 [2]. The structure is associated with a strongly increased neutral pressure, thus enabling efficient pumping. This offers the possibility for a very efficient exhaust regime in a stellarator with island divertor such as W7-X, simultaneously with significantly reduced convective heat loads onto the divertor itself. The spatial separation of the HDZ and the carbon radiation region may imply that such a state can be reached even in a non-carbon machine, and might therefore be DEMO-relevant.
\end{abstract}

Keywords: Island Divertor, Stellarator, Marfe, Volume Recombination, Particle Exhaust

PACS numbers: $52.55 . \mathrm{Hc}, 52.55 . \mathrm{Rk}$

Submitted to: Nucl. Fusion 


\section{Introduction}

Some years ago a high-density zone (HDZ) was discovered in the island divertor of the W7-AS stellarator [1]. Electron densities up to $7 * 10^{20} \mathrm{~m}^{-3}$ were measured in the upper divertor region by means of a spectrometer with lines-of-sight (LOS) parallel to the target. The HDZ was detected in a range of 0.5 to $3 \mathrm{~cm}$ from the target. Simultaneously, an intense $H_{\beta}$ emission was measured with the same spectrometer as a consequence of very strong volume recombination. Thus, the HDZ was a region with strong volume recombination. Consistent with that observation, a significant increase in the sub-divertor neutral pressure on top of the device was also measured [3].

The maximum density in the strike lines was only $3.5 * 10^{19} \mathrm{~m}^{-3}$, as measured by the Langmuir probes [4], ie. a factor of 20 below that in the HDZ nearby. Basic estimates show that the high electron density in the HDZ cannot substantially be attributed to the volumetric source due to ionization, such as seen in the so-called high-recycling regime. On the contrary, theoretical and experimental studies show that generally in W7-AS, the divertor plasma density approximately tracked the upstream density (see Fig. 8 in [5]). The physics of the HDZ is not yet understood.

We perform here a new analysis of these now more than a decade old data, determining poloidal, radial and partly the toroidal extent of the carbon radiation as well as the HDZ, thus complementing the study in [1] and making some conclusions and hypotheses based on these findings. We do this by combining several different diagnostic measurements and performing a heuristic but well founded tomographic inversion.

\section{Background information: The W7-AS divertor and the HDZ discharges}

The magnetic topology and the geometry of the island divertor are shown in Figs. 3 and 4. In the magnetic standard configuration with $\iota=5 / 9$ there are at most four strike lines on the targets. These originate from intercepting two islands. A detailed discussion of the strike line geometry can be found in [4].

The discharges where the HDZ appeared were positive density ramps and went successively through 5 phases as the density was raised, which we here label 0 through 4 as follows:

Phase 0 The divertor plasma is attached to the target.

Phase 1 The plasma is detached from the target.

Phase 2 The HDZ appears, the plasma stays detached.

Phase 3 A MARFE appears.

Phase 4 The discharge terminates due to a radiation collapse.

The physics of phases 0 and 1 was described in the W7-AS review paper [6]. The existence of the HDZ was identified by the increase of the sub-divertor neutral pressure behind the top divertor, triggered by volume recombination in the HDZ. Fig. 1 shows time traces for an example discharge (shot number 54490).

The figure shows the temporal evolution of the line-averaged density $n_{e}$, the subdivertor pressures (see Figs. 3 and 4 for the location of the pressure gauges), the energy content $W_{\text {dia }}$, the heating power of the NBI and the radiative losses. At $\mathrm{t}$ $=0.25 \mathrm{~s}$ the neutral pressure asymmetry between top and bottom divertor started 
to develop, resulting in a top-bottom ratio of 3:1. The formation of the HDZ was accompanied by a small peak of the line-averaged density and a slight reduction of the global plasma energy content, presumably due to the associated increased radiation losses.

In this paper we focus almost exclusively on phase 2 where relatively stable conditions with the HDZ were seen for several hundreds of milliseconds. More information about these discharges can be found in [7] and [6].

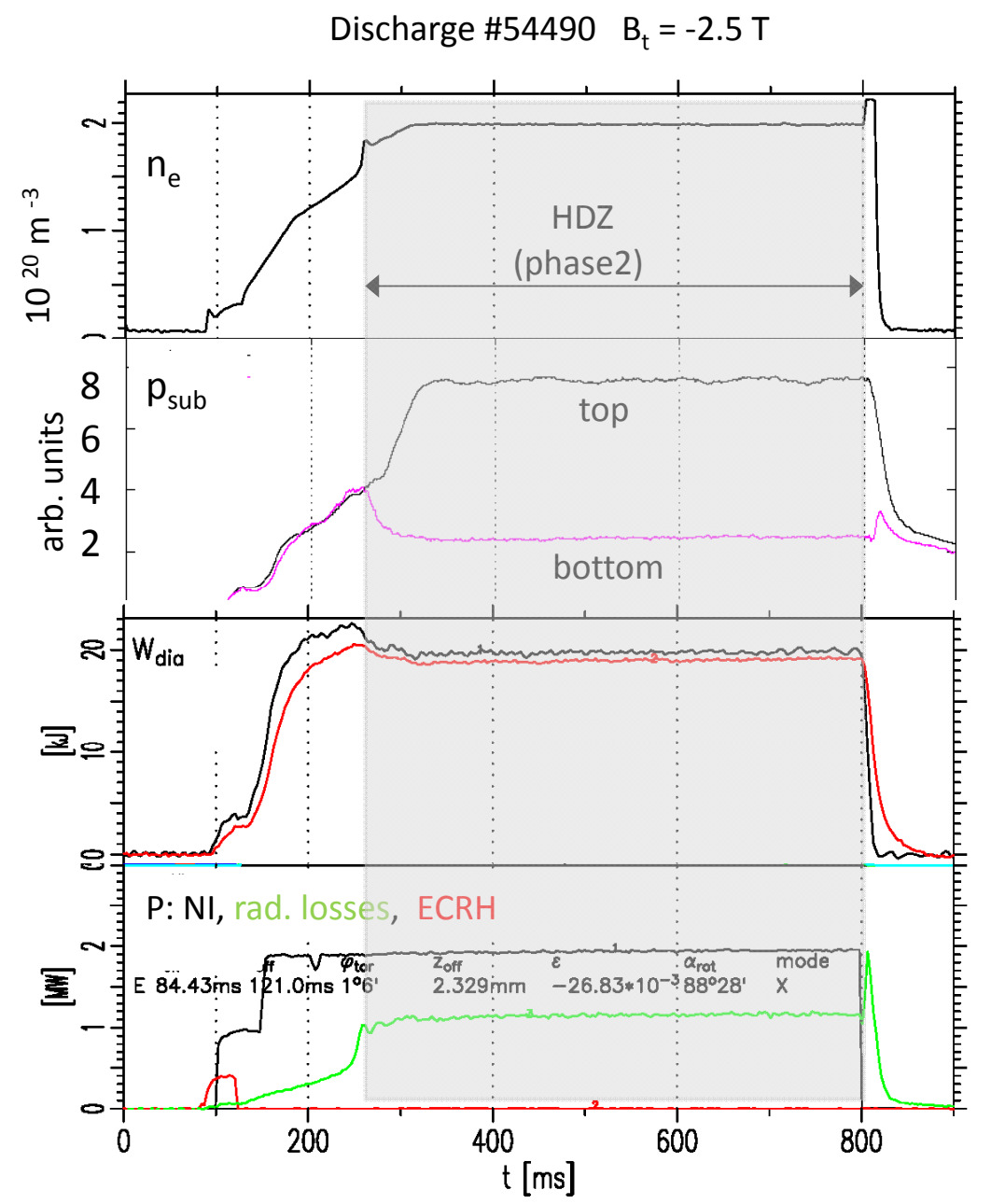

Figure 1. Discharge 54490 with HDZ - the HDZ can be easily identified by the asymmetry of the sub-divertor neutral pressures $p_{s u b}$. The figure shows the time traces of the line-averaged density, the neutral pressures (black - pressure on top, blue - pressure at bottom), the energy content (2 traces at different angles), the neutral beam heating power (black) and the total radiative losses (green). At $\mathrm{t}=$ $0.25 \mathrm{~s}$ the neutral pressure asymmetry between top and bottom divertor started to develop, resulting in a top-bottom ratio of 3:1. The neutral pressure data shown are not absolutely calibrated but the two signals for the top and bottom are directly comparable. 


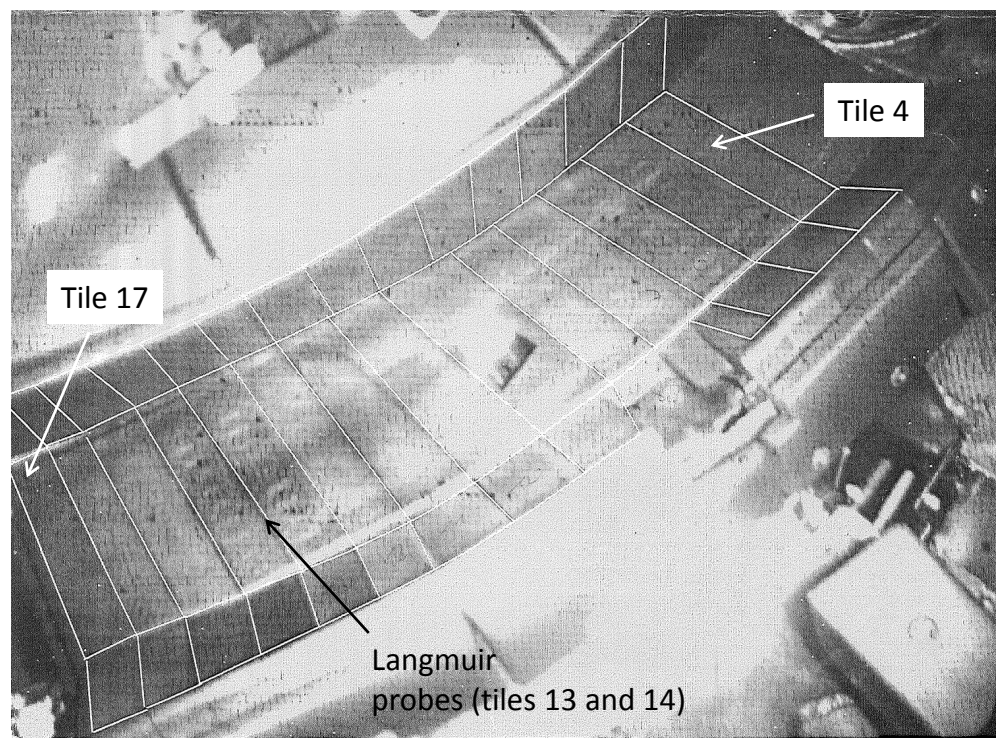

Figure 2. Image of the lower divertor showing the target tiles and the baffles. The numbering scheme for the tiles is shown by indicating target tiles 4 (right) and 17 (left).

\section{General layout of the divertor diagnostics}

Figure 2 is a picture taken with the divertor CCD camera that is also used later (Section 4). Langmuir probes were placed embedded in the divertor at tile 13 and 14. Artificially added white lines delineate the individual tiles 4-17. The upper divertor has the same structure. In the upper divertor is a cut in the outer baffle by tile 8 served to create a view for the divertor spectrometer which we will use to determine the distance of the HDZ and the carbon radiation to the divertor targets. These measurements are presented in Figure 7.

Figure 3 shows a toroidal cut of the magnetic topology including the location and numbering convention for the x-points closest to the divertor. The CCD camera view, and the placement of the subdivertor pressure gauge are also shown. Figure 4 shows a zoom-in of the divertor region at the bottom of the device with strike points numbered.

\section{Toroidal-poloidal spectrally filtered divertor observations using the divertor camera}

The CCD camera aimed at the divertor (used for Figure 2) was used in nominally identical (very reproducible) discharges with different filters, allowing a poloidaltoroidal comparison between the carbon radiation and the $H_{\gamma}$ radiation associated with the strong volume recombination in the HDZ. Figure 5 shows the CII-emission, which was characterized primarily by three well-defined and narrow bright lines which extended across the entire camera view. Figure 6 shows the $H_{\gamma}$ emission for the same view, ie. it shows the HDZ. In order to effectively compare the two pictures, the bright, thin carbon radiation lines were marked with dots (one color per stripe) in Figure 5, and these were then numerically transferred to the image in Figure 6. As 


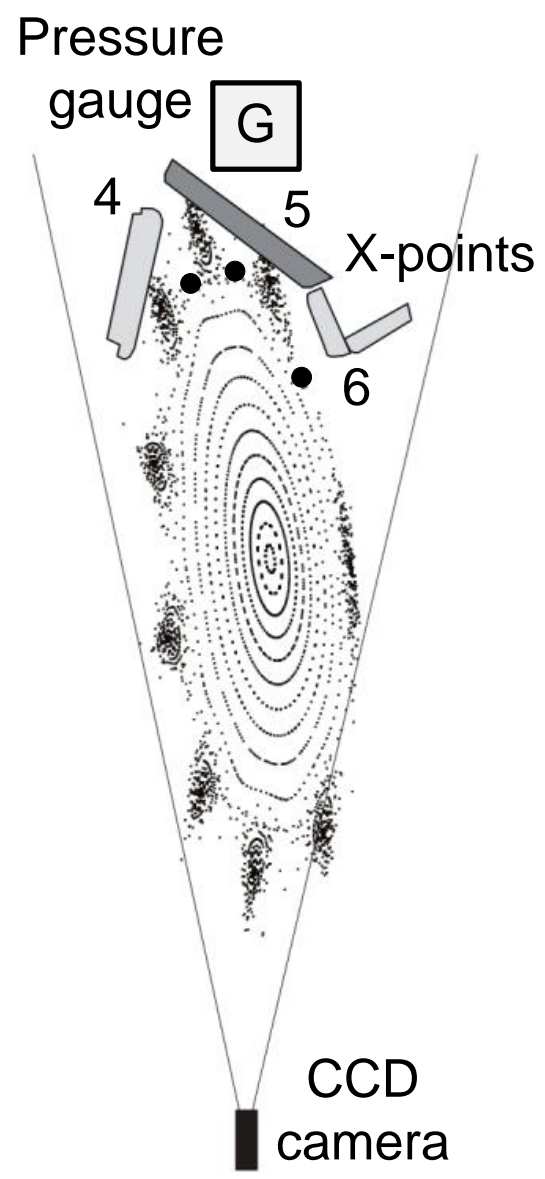

Figure 3. Magnetic geometry and viewing angle of the target plate camera viewing the divertor on top. Three X-points lie in front of the target plate. They are marked by black circles and numbered 4,5 and 6 . The island is cut two times by the upper divertor target. The position of the upper pressure gauge is also shown.

can be seen, $H_{\gamma}$ emission was of a different morphology and location than the carbon radiation. The HDZ is a single poloidally wide band of radiation that is less toroidally extended than the three carbon radiation stripes.

\section{Location of radiation relative to the divertor target plates}

The divertor spectrometer viewed parallel to the divertor target plates and was spatially resolved in the direction perpendicular to the target, allowing determination of the distance between radiation sources and the target plates. Measurements of carbon (CII) and hydrogen $\left(\mathrm{H}_{\beta}\right)$ radiation are shown in Figure 7 as a function of time. $\mathrm{H}_{\beta}$ radiation is primarily associated with volume recombination and can therefore be used to detect and localize the $\mathrm{HDZ}$, just as $\mathrm{H}_{\gamma}$ radiation. 


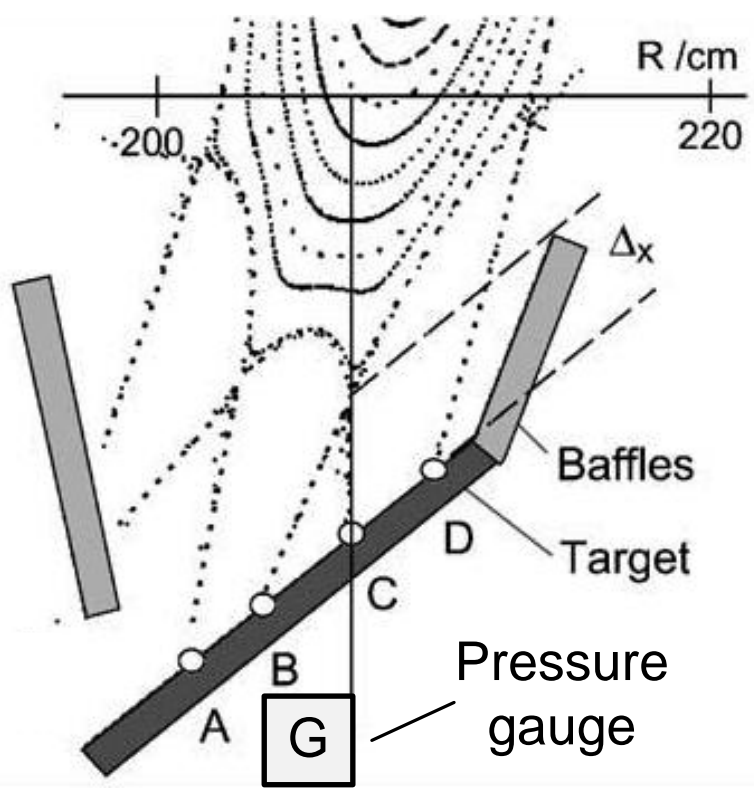

Figure 4. Magnetic geometry and strike points in the island divertor of W7-AS. The possible strike points are named with the capital letters A through D. $\Delta_{x}$ is the distance from target to X-point. The Fig. shows the divertor at bottom. The position of the pressure gauge at bottom is also shown.

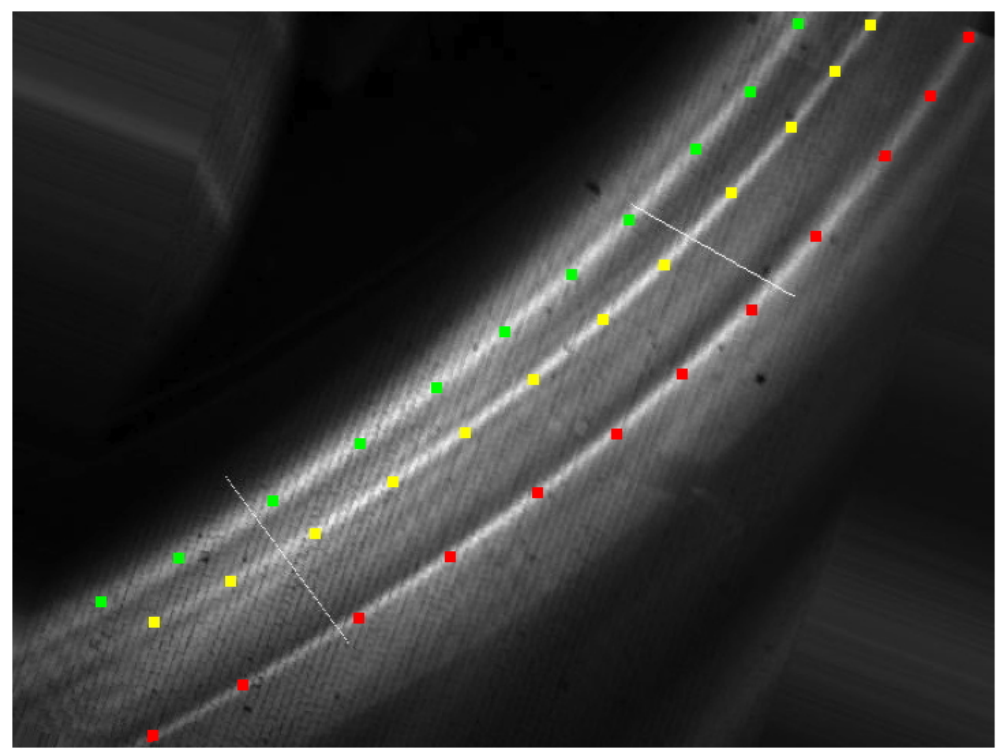

Figure 5. CII emission from the upper divertor during the HDZ (discharge 52617). The radiation was primarily concentrated in three narrow stripes. Colored marks following these three lines were added manually to the Figure - these also appear for reference in Figure 6 where the $\mathrm{H}_{\gamma}$ line radiation is shown for the same view. The target tiles 5 and 13 are marked by the white lines. 


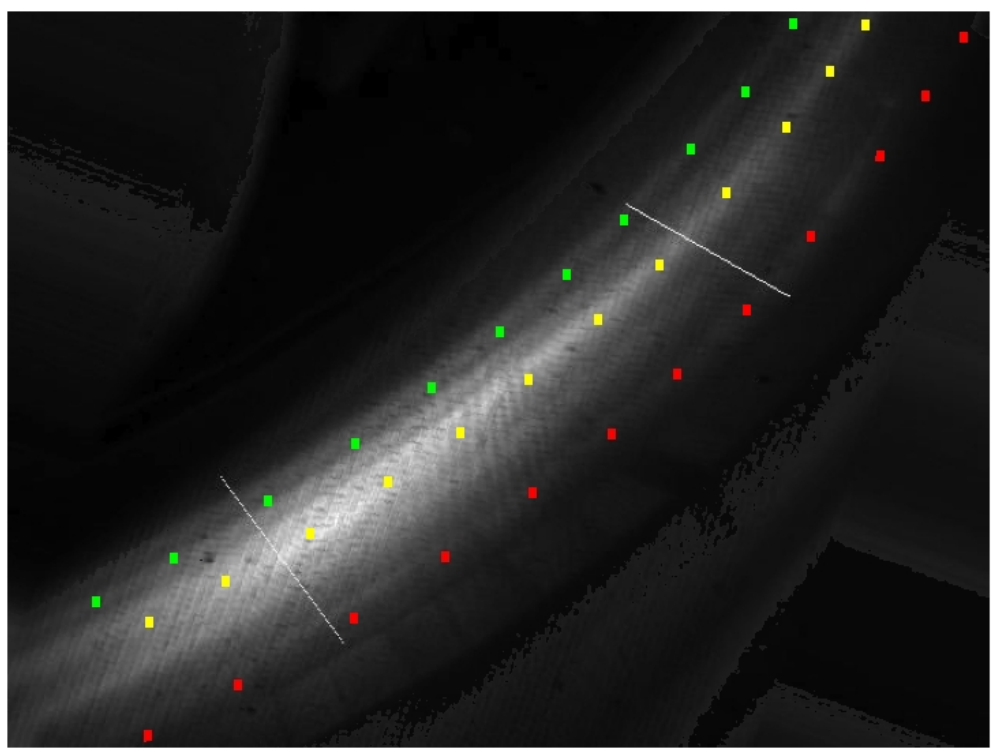

Figure 6. $H_{\gamma}$ image from the upper divertor during the HDZ (discharge 52615) using the same camera view as for Figure 5. The $H_{\gamma}$ emission is predominantly due to 3-body-recombination, and thus gives information about the poloidal and toroidal extent of the HDZ. The colored points marking the path of the carbon radiation stripes in Figure 5 are shown also in this figure to allow a comparison. It is clear that the morphology is different, with a poloidally much broader $\mathrm{H}_{\gamma}$ emission and a somewhat shorter toroidal extent.

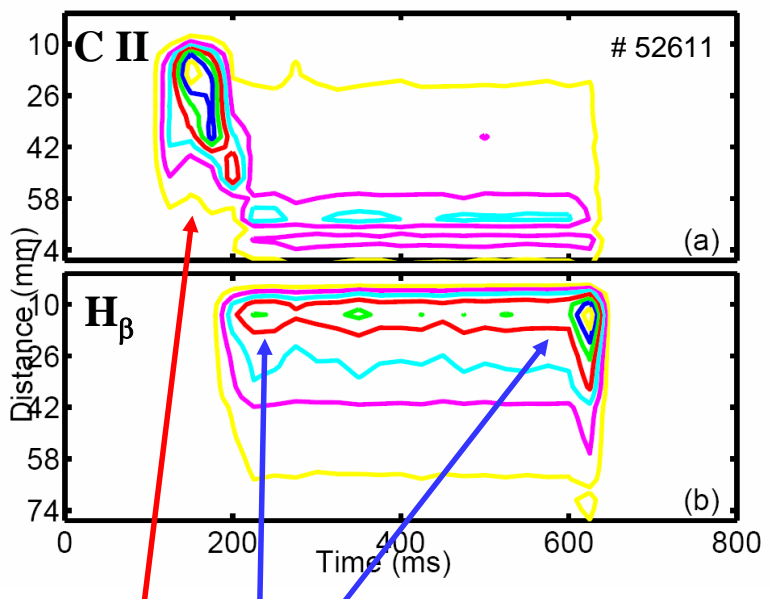

Figure 7. The temporal evolutions of the CII and $H_{\beta}$ emission in the upper divertor are measured with the divertor spectrometer. The iso-intensity contours are shown, normalized to the maximum signal observed, in the following order from low to maximum: yellow, magenta, cyan, red, green blue, yellow (again).

With increasing line-averaged density, the CII emission first gradually detaches from the target (phase 1). During phase 1, there is negligible $\mathrm{H}_{\beta}$ radiation. As the carbon radiation lifts off and moves more than $40 \mathrm{~mm}$ from the target plates, phase 2 begins: 
the HDZ appears, with a peak intensity just some $10 \mathrm{~mm}$ above the target. In phase 2 the carbon radiation resides well away from the hydrogen radiation and has two maxima, one around $60 \mathrm{~mm}$ from the target, one appr. $72 \mathrm{~mm}$ above the target. For more than $300 \mathrm{msec}$ this situation persists (over the whole flat top time of the discharge). Thus, the detachment of the carbon radiation and the appearance of the HDZ appear to be linked. The distance of the $H_{\beta}$ emission from the target was about 10 to $25 \mathrm{~mm}$, i.e. the emission from the HDZ and the carbon emission were clearly spatially separated in this view. Finally, after $\mathrm{t}=600 \mathrm{msec}$, the plasma was terminated.

The maximum density $7 * 10^{20} \mathrm{~m}^{-3}$ was measured at a distance of $1.2 \mathrm{~cm}$ from the target. The whole range of the HDZ was 0.5 to $3 \mathrm{~cm}$ from the target as measured by the divertor spectrometer. Combining several views, we identify the poloidal position of the HDZ (phase 2) with respect to the 4 strike points observed in the attached divertor plasma (phase 0$)$.

\section{Heuristic tomographic inversion}

It is clear from both the divertor camera (Section 4) and the divertor spectrometer measurements (Section 5) that the main carbon radiation is located well away from the HDZ. The measurements presented allow for a heuristic tomographic inversion to locate the HDZ and the carbon radiation (CII) poloidally and radially (with toroidal extents already quite well measured by the camera images in Section 4). We perform this in Fig. 8. The plotted magnetic surfaces are for a vacuum configuration. Finite pressure effects were not taken into account for these shots. However, the good agreement between the location of the carbon radiation and the vacuum $x$-points probably means that finite pressure effects were small. The blue lines parallel to the target bound the region of high line-integrated intensity of $H_{\beta}$ light (compare with the bottom part of Fig. 7). The blue lines drawn from the camera view bound the poloidal (along-target) extent of the high intensity $H_{\gamma}$ band. Thus, the HDZ is located at the intersection of those high-intensity lines of sight and is marked with a bluish transparent color. The red lines indicate the (very localized) high intensity carbon radiation signals from the two diagnostics. We conclude that the data strongly support that the three carbon radiation lines are located at x-point lines (marked with black circles). The divertor spectrometer sees only two maxima because the third one is outside its field of view. Note that only the CII emission was measured with crossed lines of sight. The divertor spectrometer data show that the CIII and CII emission regions closely overlap. No VUV spectrometer was available on W7-AS, so the CIV line was not measured.

\section{Reversal of the toroidal magnetic field}

For the results shown so far, the HDZ was detected in the divertor located on top of the plasma and not in the divertor at the bottom, although diagnostics there (eg. divertor neutral pressure gauges) would have been able to detect a HDZ. These results were for the "standard" magnetic field direction used in W7-AS (toroidal component clockwise when viewed from above). When the magnetic field direction was reversed (relative to the situation shown in Fig. 1 for the normal field direction), and similar discharges were run, the pressure asymmetry was reversed, too, and the HDZ appeared at the 


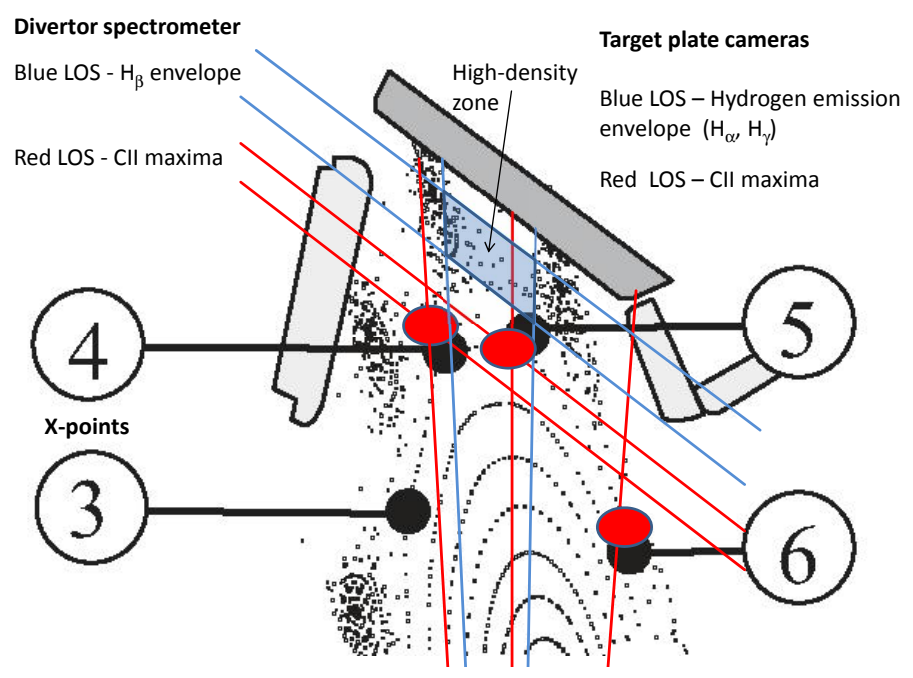

Figure 8. Schematic presentation of the radiation zones during the discharge phase with HDZ. The approximate X-point positions are shown, numbered. The data are consistent with the outermost CII emission stripe overlaying with $\mathrm{x}$-point line number 6 , which is outside the view of the divertor spectrometer. It is not clear if $\mathrm{x}$-point 3 overlaps too much with $\mathrm{x}$-point 4 to separate their radiation with the camera view, or if it simply did not radiate.

bottom divertor instead of the top divertor. Fig. 9 shows the $H_{\gamma}$ image of the target at the bottom divertor in a discharge with reversed field. It is not too dissimilar to what was observed on top for the standard direction of the magnetic field. Thus, the formation of the HDZ and/or the sustainment of it must be at least partly be caused by a magnetic drift effect and was - other than the up-down reversal- substantially similar between the standard and the reversed field direction.

\section{Discussion and conclusion}

It is useful here to compare and contrast our findings with those of MARFEs in tokamaks. MARFEs in tokamaks are mostly triggered by a thermal instability of the impurity radiation (typically carbon), and are often located at the x-point. A good example is the CIII radiation from the X-point MARFE in JET, shown in Fig. 4 in [8]. We see a stable carbon radiation near the x-points. However, the simultaneous, stable existence of the HDZ appears to be more closely related to so-called "fat" MARFEs located on the inboard side of tokamaks very close to the wall. These were not directly related to impurity radiation but appeared when the last closed flux surface was very close to the inboard wall, as seen in the TEXTOR-94 tokamak [2].

A theoretical model for a condensation process in hydrogen was published by Tokar [9]. This model had solutions that were stable as well as ones that were unstable. What we see here is a situation where the transition into the HDZ is very fast and presumably hysteretic, implying that the plasma (edge) jumps from one stable operating point to another. One can consider this as a complete transition from attached to detached plasma in the localized region of the HDZ. In the tokamak situation, the existence of a wall very close to the last closed flux surface appears to be a prerequisite for the 


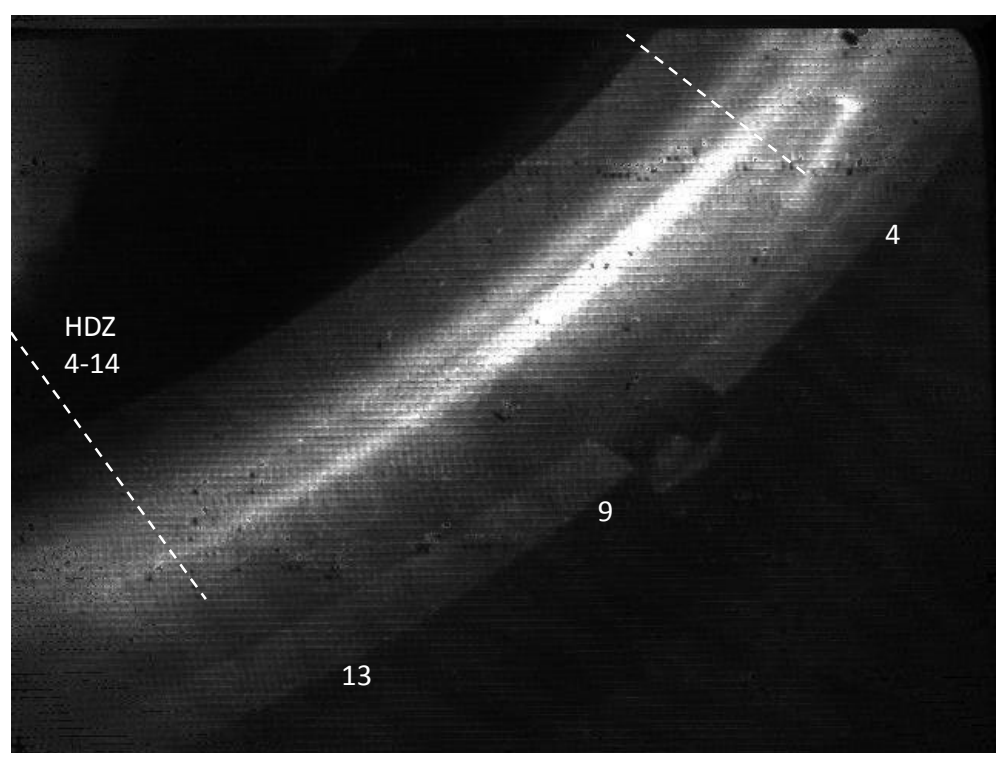

Figure 9. The $H_{\gamma}$ emission in front of the target at bottom with reversed magnetic field. The emission pattern shows the approximate extension of the HDZ. It followed the direction of the target and, thus, the helical direction. This approximate range of the HDZ is indicated by the dotted white lines. The orientation of the target is the same as in Fig. 6

"fat" MARFE. This is clearly not the case for the W7-AS results reported here. Here, the HDZ does appear near the wall but the x-points are well away from the target plates. The proximity of the HDZ to the wall strongly suggests that wall-recycling is a key ingredient to the hydrogen condensation, as predicted by Tokar [10].

The advantages of this operation mode are clear: Stable detachment with high pumping efficiency and very low divertor heat loads and low sputtering would be a very attractive divertor operation mode for a tokamak or a stellarator, including W7-X.

Since the carbon radiation is well away from the HDZ, the question arises if one could access and sustain this operation mode, even if there were no carbon radiation, eg. in a carbon-free machine, as a D-T reactor must be. This is, however, quite speculative since strong carbon radiation is present near the target before the appearance of the $\mathrm{HDZ}$ in that region, and remains near the x-point during the HDZ phase (phase 2), potentially "pre-cooling" the plasma as it flows across the x-point region, before it reaches the HDZ.

Even if some edge impurity radiation losses turn out to be needed, those might be achieved in a carbon-free machine by using the radiating mantle techniques with eg. nitrogen developed at ASDEX-Upgrade [11].

These and other questions can hopefully be addressed in W7-X. The magnetic geometry of the island divertor in the W7-X stellarator should also be favorable for this condensation process. The long connection lengths may allow the plasma to cool down sufficiently on its way from upstream to the target plates to initiate the condensation once it reaches the target plates. Configurations 
with different values for the distance between x-point and divertor target can be tested. In W7-X, the distance between x-point and target can be varied between 0 and $20 \mathrm{~cm}$. As an example, the so-called low mirror configuration has a target to x-point distance of $6 \mathrm{~cm}$, comparable to W7-AS. In the low shear configuration the distance is $14.6 \mathrm{~cm}$, probably too large for a condensation. The planned divertor diagnostics for W7-X include a sophisticated visible observation endoscope system, several helium beams, divertor-integrated Langmuir probes and neutral pressure gauges and should enable detailed studies of this and other divertor physics phenomena.

\section{Acknowledgments}

The authors would like to thank Prof. Tokar, Prof. Reiter and Dr. Kotov of FZ Jülich for valuable discussions about the condensation process in a pure hydrogen plasma. Furthermore, they thank for the fruitful discussion with Dr. Loarte from ITER and Prof. Schmitz from University of Wisconsin-Madison. The authors would also like to thank H. Hölbe for his investigations of the magnetic configurations of W7-X.

\section{References}

[1] N. Ramasubramanian, R. König, Y. Feng, L. Giannone, P. Grigull, T. Klinger, K. McCormick, H. Thomsen, and U. Wenzel. Characterization of the island divertor plasma of W7-AS stellarator in the deeply detached state with volume recombination. Nucl. Fusion, 44:992-998, 2004.

[2] U. Samm, M. Brix, F. Durodie, M. Lehnen, A. Pospieszczyk, J. Rapp, G. Sergienko, B. Schweer, M.Z. Tokar, and B. Unterberg. MARFE feedback experiments on TEXTOR-94. J. Nucl. Mat., 266-269:666, 1999.

[3] K. McCormick, P. Grigull, R. Burhenn, H. Ehmler, Y. Feng, L. Giannone, G. Haas, and F. Sardei. Neutral pressure behavior for diverted discharges in the Wendelstein 7-AS Stellarator. $J$. Nuclear Mat., 337-339:520-524, 2005.

[4] P. Grigull, K. McCormick, Y. Feng, A. Werner, R. R. Brakel, H. Ehmler, F. Gadelmeier, D. Hartmann, D. Hildebrandt, R. Jaenicke, J. Kisslinger, T. Klinger, R. König, D. Naujoks, H. Niedermeyer, N. Ramasubramanian, F. Sardei, F. Wagner, and U. Wenzel. Influence of magnetic field configurations on divertor plasma parameters in the w7-as stellarator. $J$. Nuclear Mat., 312-316:1287-1291, 2003.

[5] Y. Feng, F. Sardei, P. Grigull, K. McCormick, J. Kisslinger, and D. Reiter. Physics of island divertors as highlighted by the example of W7-AS. Nucl. Fusion, 46:807-819, 2006.

[6] M. Hirsch, J. Baldzuhn, C. Beidler, J. Kisslinger, and et al. Major results from the stellarator Wendelstein 7-AS. Plasma Phys. Control. Fusion, 50:1-204, 2008.

[7] U. Wenzel and M. Goto. Estimating the plasma flow in a recombining plasma from the $H_{\alpha}$ emission. J. Nucl. Mat., 415:S1170-S1173, 2011.

[8] J. Rapp, W. Fundamenski, L.C. Ingesson, S. Jachmich, A. Huber, and et al. Septum assessment of the JET gas box divertor. Plasma Phys. Control. Fusion, 50:1-30, 2008.

[9] M.Z. Tokar. Ionization Instability of the Tokamak Edge Plasma with Particle Recycling from the Wall. Physica Scripta, 31:411-414, 1985.

[10] M.Z. Tokar, J. Rapp, D. Reiser, U. Samm, F.C. Schüller, G. Sergienko, and P.C. de Vries. Localized recycling as a trigger of MARFE. J. Nucl. Mat., 266-269:958-962, 1999.

[11] A. Kallenbach, R Dux, J. C. Fuchs, R Fischer, B Geiger, L Giannone, Herrmann A, T Lunt, V Mertens, R McDermott, R Neu, T Pütterich, S Rathgeber, V Rohde, K Schmid, J Schweinzer, Treutterer W, and ASDEX Upgrade Team. Divertor power load feedback with nitrogen seeding in ASDEX Upgrade. Plasma Phys. Controlled Fusion, 52(5):055002, 2010. 